\title{
Observation of dynamical crater-shaped charge distribution in the space-time imaging of monolayer graphene
}

Jongweon Cho, ${ }^{\text {a,b }}$ Jau Tang, ${ }^{\text {a,c }}$ Taek Yong Hwang ${ }^{\mathrm{a}, \mathrm{d}}$ and Ahmed H. Zewail ${ }^{\text {ta }}$

aPhysical Biology Center for Ultrafast Science and Technology, Arthur Amos Noyes Laboratory of Chemical Physics, California Institute of Technology, Pasadena, California 91125, United States

${ }^{\mathrm{b}}$ Department of Physics, Myongji University, Yongin 17058, Korea

${ }^{\mathrm{c}}$ The Institute of Technological Sciences, Wuhan University, Wuhan 0027, China

${ }^{\mathrm{d}}$ Molds \& Dies R\&D Group, Korea Institute of Industrial Technology, Bucheon 14441, Korea

*Corresponding authors: jwcho@mju.ac.kr, jautang@caltech.edu

†Deceased

Supplementary Text

Figures S1 to S9

Table S1

References 


\section{Supplementary Text}

$\underline{\text { Two pump-pulse experiments }}$

We note here that Fig. S4 presents our systematic control of two pump-pulse experiments and Figs. S4(d-f) display the key results. Fig. S4(d) shows a peanut-shaped contour instead of two crossed crater-like distributions when the two pump beams are slightly separated in space and temporally overlapped. Each pump produces a crater shape at $80 \mu \mathrm{J} / \mathrm{cm}^{2}$ as shown in Figs. S4(b-c). Fig. S4(e) shows a crater-shaped profile when the two beams are spatially overlapped and temporally delayed by 13 ps. Lower fluence is used here since at this value a single-lobed profile is retained for each pump pulse (with a signal-to-noise ratio optimized for the measurements). Note that the crater shape is still present in Fig. S4(e), as can be clearly seen in Fig. S4(f), the cross section along a stripe shown in Fig. S4(e). Because Rabi flopping is a coherent, resonant optical process and the carriers dephasing time in graphene is expected to be far less than $13 \mathrm{ps},{ }^{1}$ if the crater-like distribution arises due to Rabi oscillations, we should have seen a single-lobed distribution, not a crater shape as observed, at 13 ps [Fig. S4(e)].

$\underline{\text { Theoretical modeling for carrier photoexcitation }}$

To model the observed crater-shaped dynamic behavior at relatively short times we introduced the following kinetic exciton model involving three states as illustrated in Fig. S5:

$$
\begin{aligned}
& \frac{\partial f_{2}(x, y, t)}{\partial t}=w(x, y) H(\tau-t) f_{1}(x, y, t)-\alpha f_{2}(x, y, t)-\beta f_{2}(x, y, t), \\
& \frac{\partial f_{1}(x, y, t)}{\partial t}=w(x, y) H(\tau-t) f_{0}(x, y, t)-w(x, y) H(\tau-t) f_{1}(x, y, t)+\alpha f_{2}(x, y, t), \\
& \frac{\partial f_{0}(x, y, t)}{\partial t}=-w(x, y) H(\tau-t) f_{0}(x, y, t)+\beta f_{2}(x, y, t),
\end{aligned}
$$


where $f_{0}(x, y, t), f_{1}(x, y, t)$, and $f_{2}(x, y, t)$ represent the time-dependent spatial distribution of the population on the ground state, one e-h pair state, and two e-h pair state, respectively. The much slower e-h recombination (rate constant $\gamma$ ) is neglected here, but can be included later assuming an exponential decay $\exp (-\gamma t)$. Here, $w(x, y)$ denotes an optically induced pumping rate with spatial dependence having the Gaussian-shaped laser intensity profile. To describe the time dependence of the pumping action from an excitation laser pulse, we consider for simplicity a rectangular-shaped pulse for the temporal profile. $H(t)$ is the Heaviside step function and $\tau$ is the laser excitation period (pulse width used in the experiment). Since we are primarily concerned with the crater-shaped dynamic behavior which occurs within a few ps, such behavior could be simulated effectively by solving numerically the above differential equations.

Based on our experimental observation the dynamic image intensity follows a delayed quasiexponential growth with a rise time of $\sim 56$ ps (see Fig. S3(b) and Fig. 3(d) in main text), implying, as expected, that the SEM signal does not come from the neutral excitons which are produced immediately after the initial laser pump excitation, but rather from the charge separated electrons and holes as the excitons break apart. The formation of neutrally charged photoexcited e-h pairs remain elusive to USEM detection until they undergo such charge separation.

The short-time dynamics regarding the excitation and relaxation of e-h pairs is governed by the above rate equations which give rise to crater-shaped U-SEM signals. The growth of the dynamic image intensity can then be described phenomenologically using $\left(f_{1}+2 f_{2}\right)\left[1-\exp \left(-t / \tau_{e x}\right)\right] \exp (-\gamma t)$, where $f_{1}+2 f_{2}$ is obtained from Eq. (1) at a time around 2 ps (much greater than $1 / \beta$ ) for which $f_{1}+2 f_{2}$ reaches a quasi-steady state, with a time constant $\tau_{e x}(\sim 56 \mathrm{ps})$ which characterizes the rise of SEM signal due to charge separation between e-h pairs; $\gamma$ is the e-h recombination rate at longer times. Note that the factor of 2 for $f_{2}$ is reflective of two pairs in this state. Table $\mathrm{S} 1$ lists the parameters used in our model. Among these parameters $\beta$ and $\alpha$ are the crucial factors controlling the formation of the cratershaped U-SEM features. Here we remark that $\beta$ is a much faster process than $\alpha$, since the rates reflect 
the electron and hole density dependence in Auger processes. Our kinetic model involving a relaxation pathway from the two e-h pair state to the single e-h pair state (i.e., $\beta=0$ in our model) is able to reproduce the flat-top U-SEM spatial profile obtainable using a conventional rate equation involving the carrier density-dependent Auger recombination.

Figure S6 displays the results of fluence-dependent spatial intensity profiles with varying $\alpha$ and $\beta$. As can be seen in Fig. S6(a)-(b), the conventional Auger recombination alone (i.e., $\beta=0$ ) would only create a flat-top spatial distribution of U-SEM image contrast but could not lead to a central dip in the spatial density distribution of e-h pairs [see Fig. S6(b), as compared to Fig. S6(a)]. Moreover, as shown in Fig. S6(c)-(d), $\beta$ plays a decisive role in the formation and fluence-dependent behavior of such cratershaped features [Fig. S6(c)], whereas $\alpha$ plays a minor role [Fig. S6(d)].

To help further elucidate how fluence and the key decay rate parameter $\beta$ affect the U-SEM image intensity and the crater shape, we performed several systematic simulations. Both maximum excitation fluence (which starts to yield an intensity dip at center spots) and integrated intensities exhibit a phenomenological, simple exponential behavior with $\beta$ [Fig. S7(a)] and fluence [Fig. S7(b)], respectively.

Figure S8 shows the temporal evolution of the intensities integrated within the field of view of the U-SEM images for the excitation fluences of 3 and $60 \mu \mathrm{J} / \mathrm{cm}^{2}$. The simulated intensities capture the key temporal behavior in Fig. 3(d) as observed from the experiments.

We remark in passing that while any non-linear decay term (without involving a two e-h pairs state) faster than a linear term within the framework of our developed model may be invoked to explain the crater-like carrier profile, the auger processes are the physically leading non-linear processes that involve minimum two e-h pairs. As such, we first considered the conventional process, which yielded not the crater shape (what was experimentally observed) but the flat-top profile. Eventually, it was nonconventional Auger-assisted processes (which involve emission of phonons and plasmons) that was able to reproduce our observations. We believe the even higher-order physical processes involving more than two e-h pairs won't be physically significant owing to (semi)metallic screening of Coulomb interaction 
in graphene, substantially reducing their mutual Coulomb interactions (see, for example, ref. 2). For the sake of simplicity screening effect is neglected in our model.

\section{$\underline{\text { Carrier diffusion }}$}

The spatial expansion of U-SEM image observed experimentally in Fig. 2(b)-(c) could be modeled based on an isotropic $2 \mathrm{D}$ diffusion mechanism. To simulate the increase of the second moment of the expanding SEM images along the long elliptic axis we only need to consider 1D diffusion. According to Einstein's random walk model, the second moment increases in time $t$ as $2 D_{0} t$. For the case with a conventional time-independent diffusion constant, the resultant carrier spatial distribution is given by

$$
F(x, t)=\int_{-\infty}^{\infty} d x^{\prime} F\left(x^{\prime}\right) G\left(x, x^{\prime} ; t\right)=\int_{-\infty}^{\infty} d x^{\prime} F\left(x^{\prime}\right) \frac{1}{\sqrt{4 \pi D t}} \exp \left(-\frac{\left(x-x^{\prime}\right)^{2}}{4 D t}\right)
$$

where $G\left(x, x^{\prime} ; t\right)$ is the Greens function and $F(x)$ is the initial spatial distribution before diffusion takes place.

As shown in Fig. 3(c), the experimental data indeed exhibits a linear expansion at short times. However, flattening behavior occurs at much later times $(>500 \mathrm{ps})$. Therefore, we need to consider a scenario with a time-dependent diffusion constant $D(t)$ whose process slows down exponentially as $D_{0} \exp \left(-t / \tau_{D}\right)$ with a time constant $\tau_{D}$. However, for such a time-dependent diffusion process the resultant spatial carrier distribution involves the convolution of the time-retarded Greens function kernel $G\left(x, x^{\prime} ; \tau\right)$ given below,

$$
G\left(x, x^{\prime} ; \tau\right)=\frac{1}{\sqrt{4 \pi D_{0} \tau_{D}\left(1-e^{-\tau / \tau_{D}}\right)}} \exp \left(-\frac{\left(x-x^{\prime}\right)^{2}}{4 D_{0} \tau_{D}\left(1-e^{-\tau / \tau_{D}}\right)}\right)
$$


For a time-dependent source $F(x, t)$ that involves a SEM signal rise time of $\tau_{e x}(\sim 56 \mathrm{ps})$ as given earlier from a quasi-steady spatial distribution of $\left(f_{1}(x)+2 f_{2}(x)\right)\left[1-\exp \left(-t / \tau_{e x}\right)\right]$, with the solution to Eq. (1), the resultant distribution is given by

$$
F(x, t)=\int_{-\infty}^{\infty} d x^{\prime} G\left(x, x^{\prime} ; t\right)\left[f_{1}\left(x^{\prime}\right)+2 f_{2}\left(x^{\prime}\right)\right]+\int_{-\infty}^{\infty} d x^{\prime}\left[f_{1}\left(x^{\prime}\right)+2 f_{2}\left(x^{\prime}\right)\right] \int_{0}^{t} d \tau G\left(x, x^{\prime} ; \tau\right) \exp \left(-(t-\tau) / \tau_{e x}\right) / \tau_{e x} .
$$

Another factor involving a much slower decay with $\gamma$ can be neglected here. As illustrated in Fig. S9, the simulated temporal behavior of the second moment along the long axis could be well-fitted phenomenologically by $2 D_{0} \tau_{D}\left[1-\exp \left(-t / \tau_{D}\right)\right]$ which gives rise to a linear increase with a slope of $2 D_{0}$, with $D_{0}\left(\sim 6500 \mathrm{~cm}^{2} / \mathrm{s}\right)$ being an experimentally determined diffusion coefficient, in the shorttime limit. 
(a)

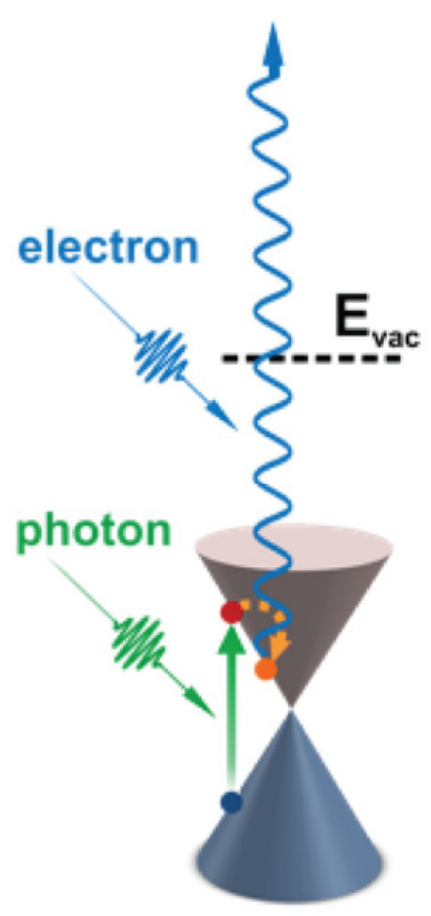

UEM-2 green power controlled by doubling crystal tuning

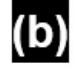

$275 \mathrm{~mW}$

$56 \mu \mathrm{m} \times 45 \mu \mathrm{m}$

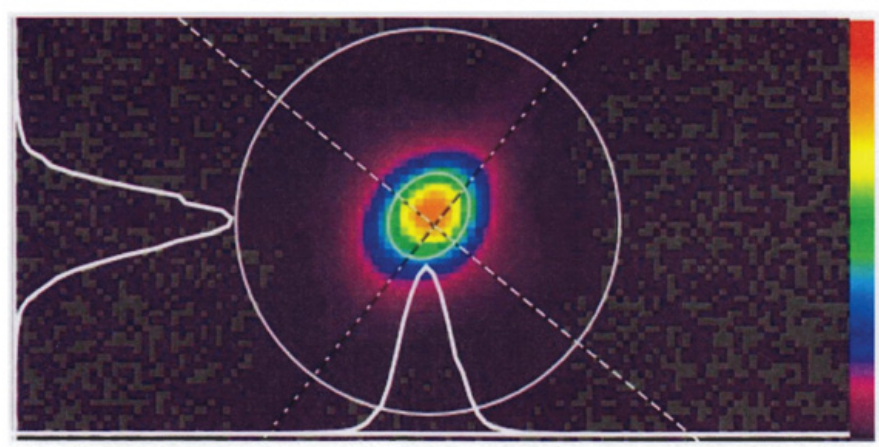

$39 \mathrm{~mW}$

$63 \mu \mathrm{m} \times 63 \mu \mathrm{m}$

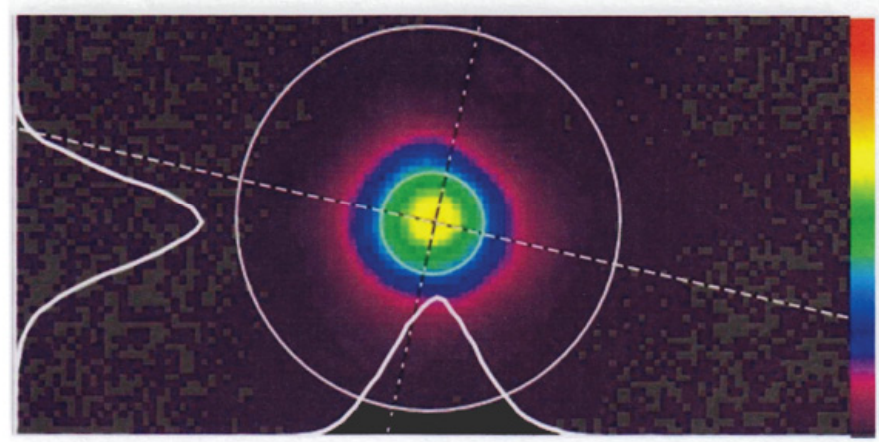

$<1 \mathrm{~mW}$

$71 \mu \mathrm{m} \times 59 \mu \mathrm{m}$

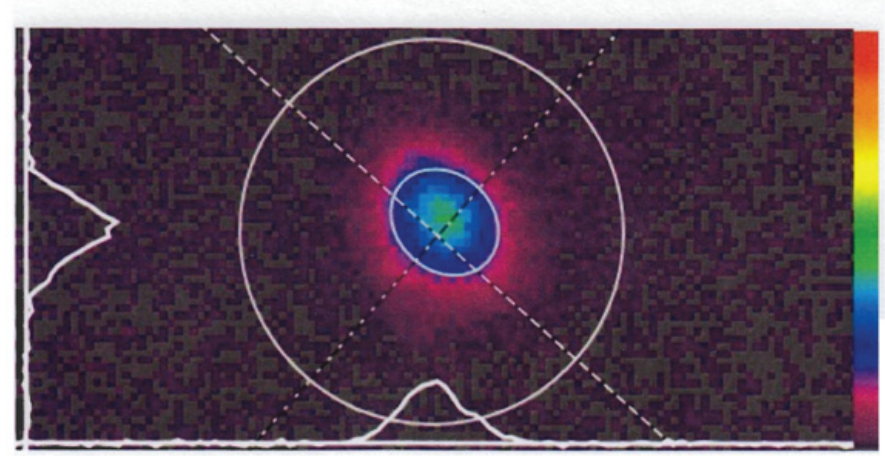

Fig. S1 Scheme of carrier excitation in graphene and laser beam shape. (a) Schematic of excitation and electron liberation. In the case of graphene, the optical excitation results in transient population of e-h pairs, and the spatial U-SEM image contrast patterns reflect the relaxation pathways of such e-h pairs. One of such dynamical events, for instance, is a single-particle intraband relaxation of a hot electron. $E_{\text {vac }}$ is the vacuum level. (b) Fluence dependence of laser beam shape. 

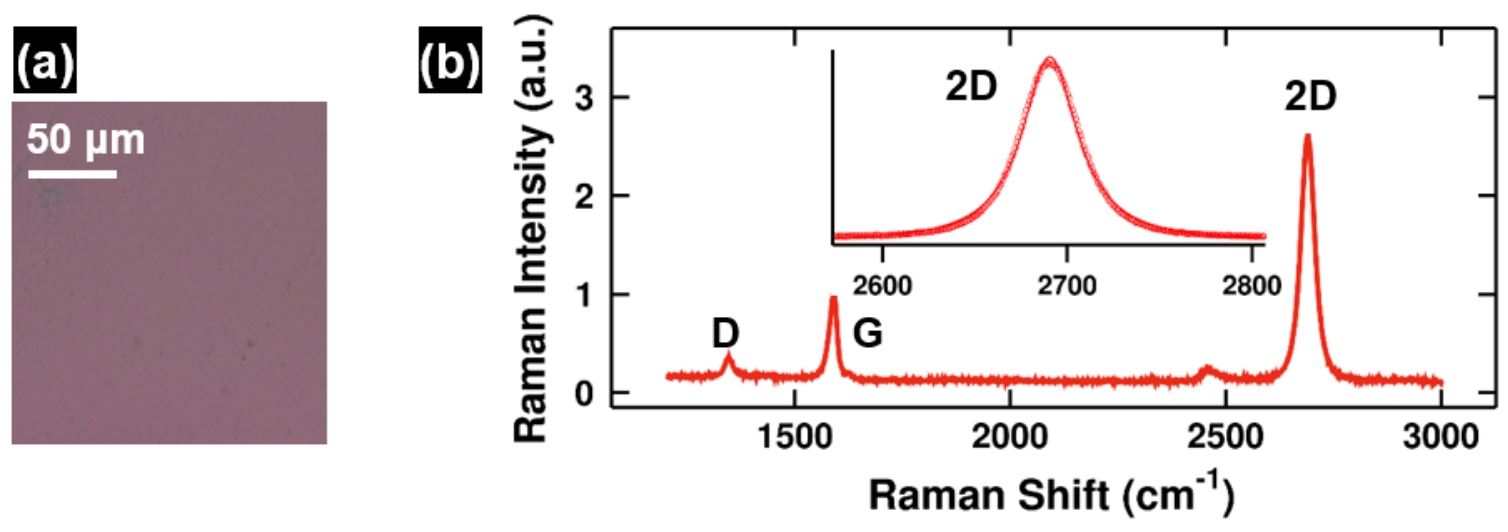

Fig. S2 Sample characterization. (a) Optical micrograph of the graphene sample used in our experiments. (b) Typical Raman spectra acquired on the investigated graphene/SiO 2 samples. The spectra indicates the presence of D-, G-, and 2D-peaks at 1346, 1589, and $2690 \mathrm{~cm}^{-1}$, respectively. The inset shows a close-up of the Raman spectra (open circle) near 2D-peak with a Lorentzian fit (solid line), suggesting the sample is monolayer graphene. ${ }^{3-5}$ 
(a) $\mathrm{Si}(100)$

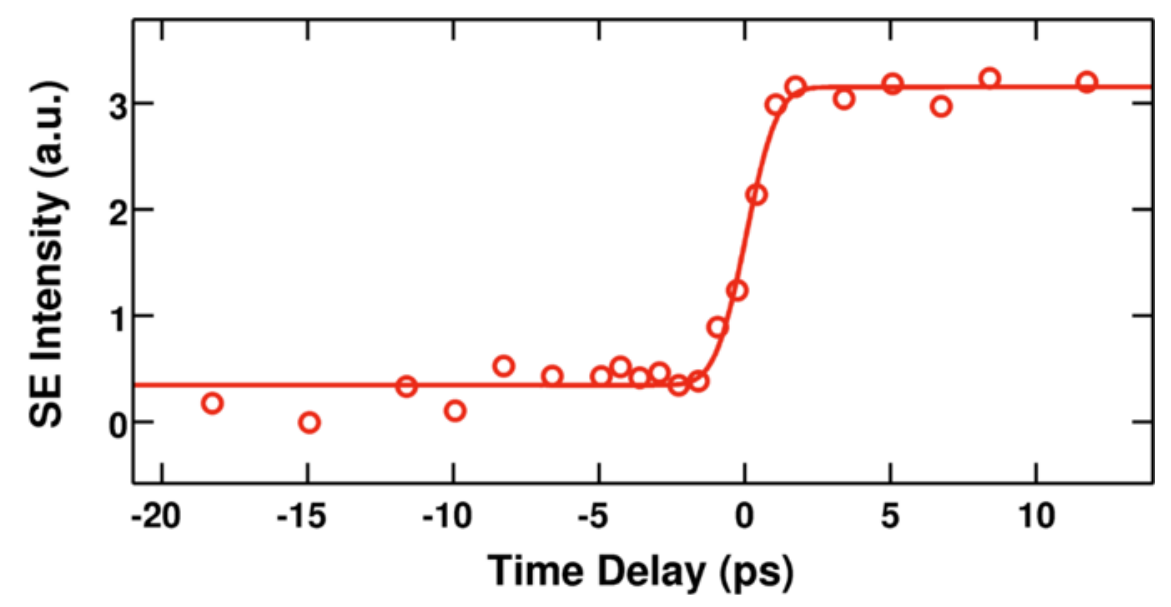

(b) Graphene

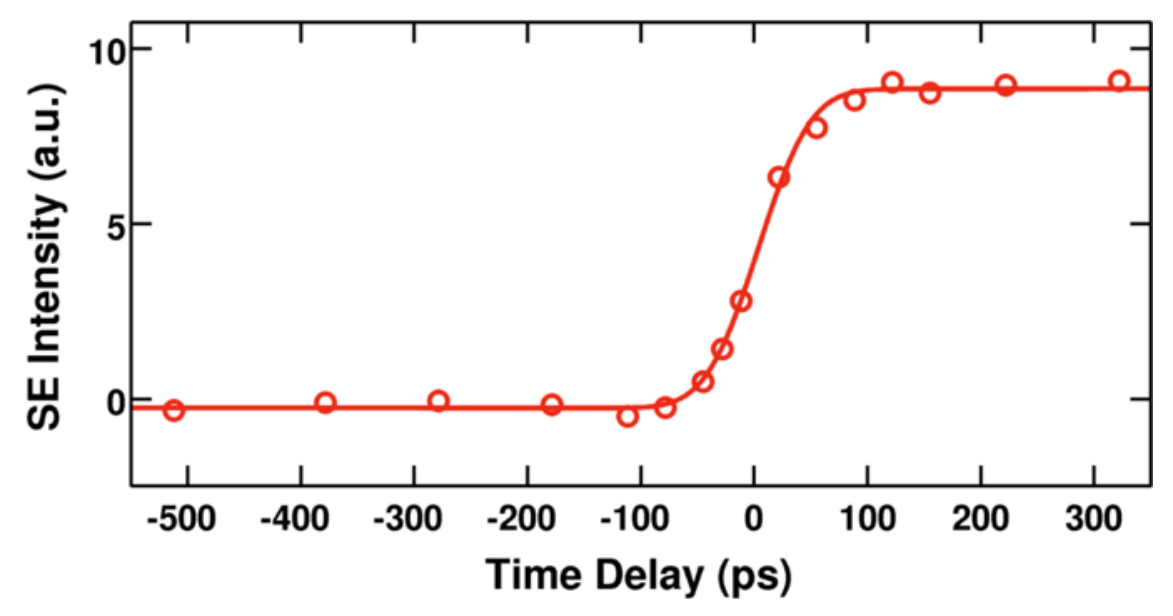

Fig. S3 Temporal evolution of U-SEM image contrast acquired at the center of laser excitation. The experimental conditions were kept identical except for the pump laser fluence. The second harmonic (photon energy of $2.41 \mathrm{eV}$ ) is used for electron pulse generation. (a) p-type $\mathrm{Si}(100)$ at the pump laser fluence of $\sim 0.6 \mathrm{~mJ} / \mathrm{cm}^{2}$. The extracted rise time is $1.2 \pm 0.2 \mathrm{ps}$. (b) Monolayer graphene at the pump fluence of $3 \mu \mathrm{J} / \mathrm{cm}^{2}$. The extracted rise time is $52 \pm 3 \mathrm{ps}$. The solid lines are sigmoidal fits to the data. 

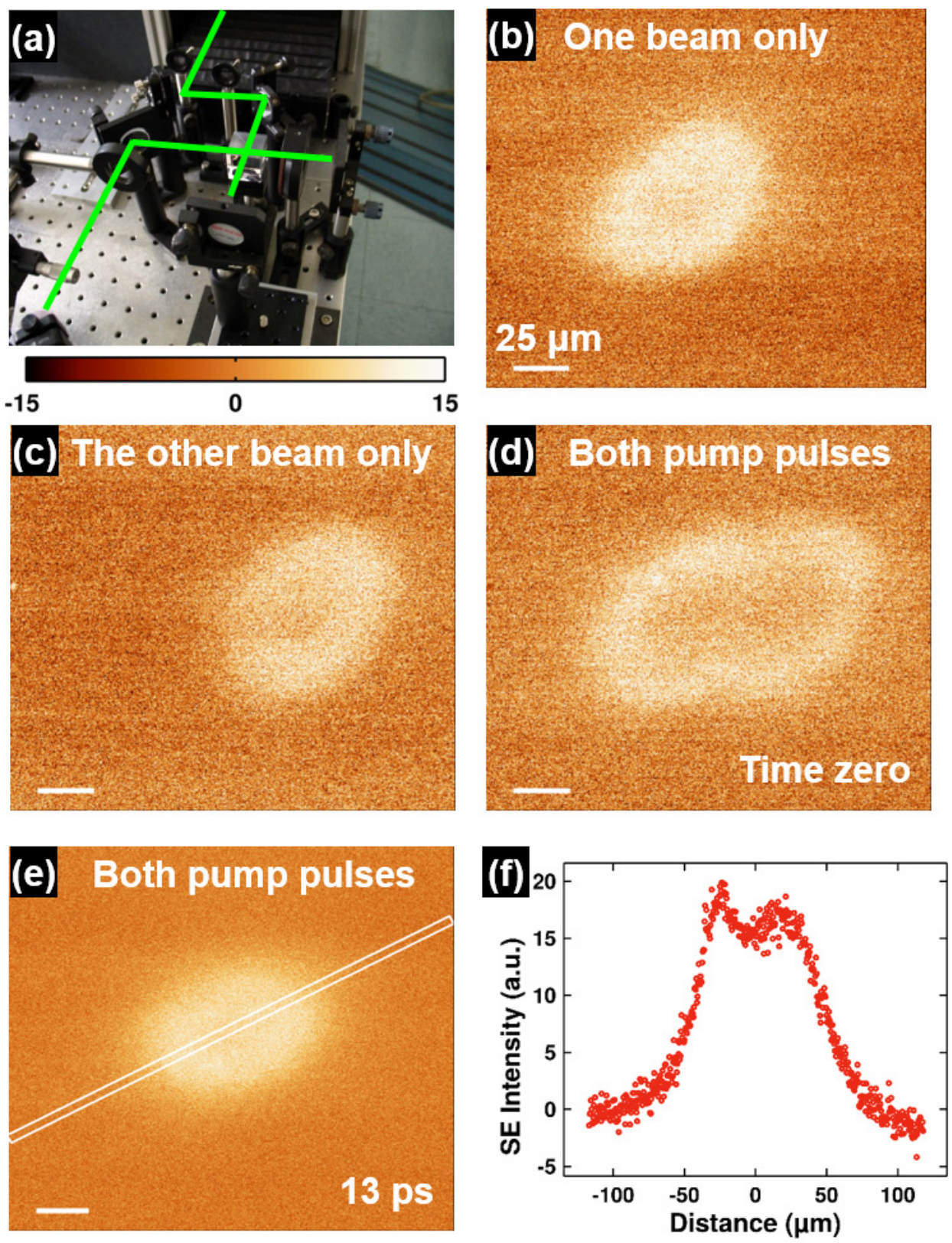

Fig. S4 Two pump-pulse experiments. (a) Photograph of Michelson interferometer configuration implemented to our apparatus. (b-e) U-SEM image (b) when only one of two pump-pulse impinges onto monolayer graphene, (c) when the other of two pump-pulse impinges onto the sample, (d) when both two (spatially separated) pump-pulses impinge onto the sample, at zero time delay $\left(80 \mu \mathrm{J} / \mathrm{cm}^{2}\right)$, (e) when both two (spatially overlapped) pump-pulses impinge onto the sample at a time delay of 13 ps (29 $\mu \mathrm{J} / \mathrm{cm}^{2}$ ). (f) Line profile along a stripe shown in Fig. S4(e), indicating a crater-shaped feature. Rabi flopping would give rise to a single-lobed feature at 13 ps at this fluence, which is in contrast to what was observed in the experiments. 
(a) Low Excitation Fluence

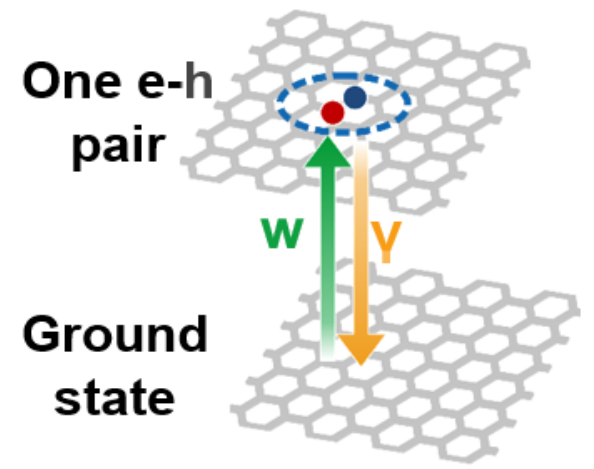

\section{(b) High Excitation Fluence}

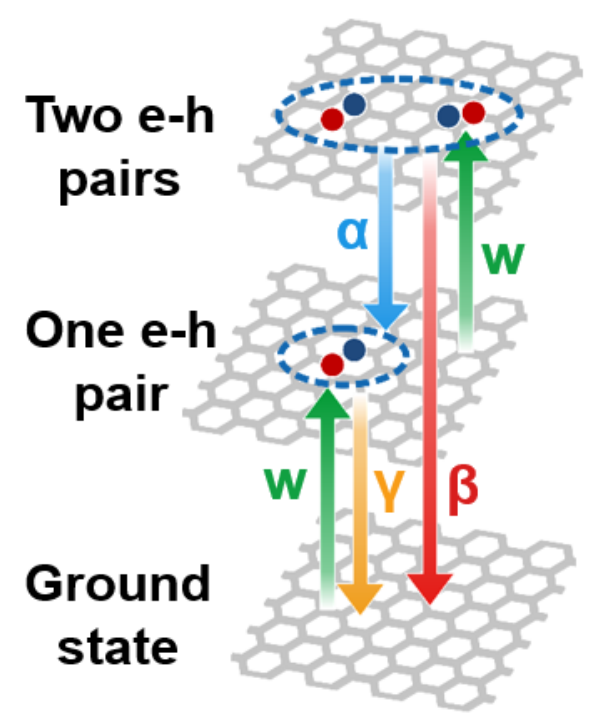

Fig. S5 Schematic diagram of e-h pairs excitation in graphene. (a) Low-fluence excitation regime. The proposed simple kinetic scheme suffices to describe the carrier dynamics resulting in a simple singlelobed contrast shape; $\mathrm{w}$ and $\gamma$ are, respectively, the rates of optical pumping and recombination from one e-h pair state to ground state. (b) High-fluence excitation regime. Here, spatial excitation of e-h pairs is illustrated by a three-level kinetic scheme involving the ground state, one e-h pair state, and a two e-h pair state; $\alpha$ and $\beta$ are, respectively, the rates of conventional Auger recombination and Augerassisted direct transition from two e-h pair state to ground state. 


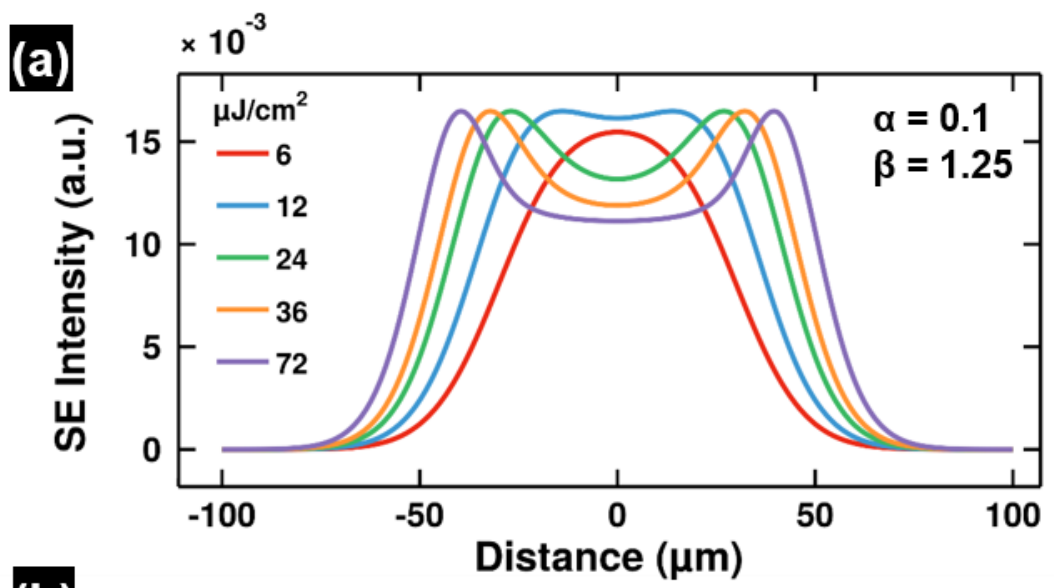

(b)
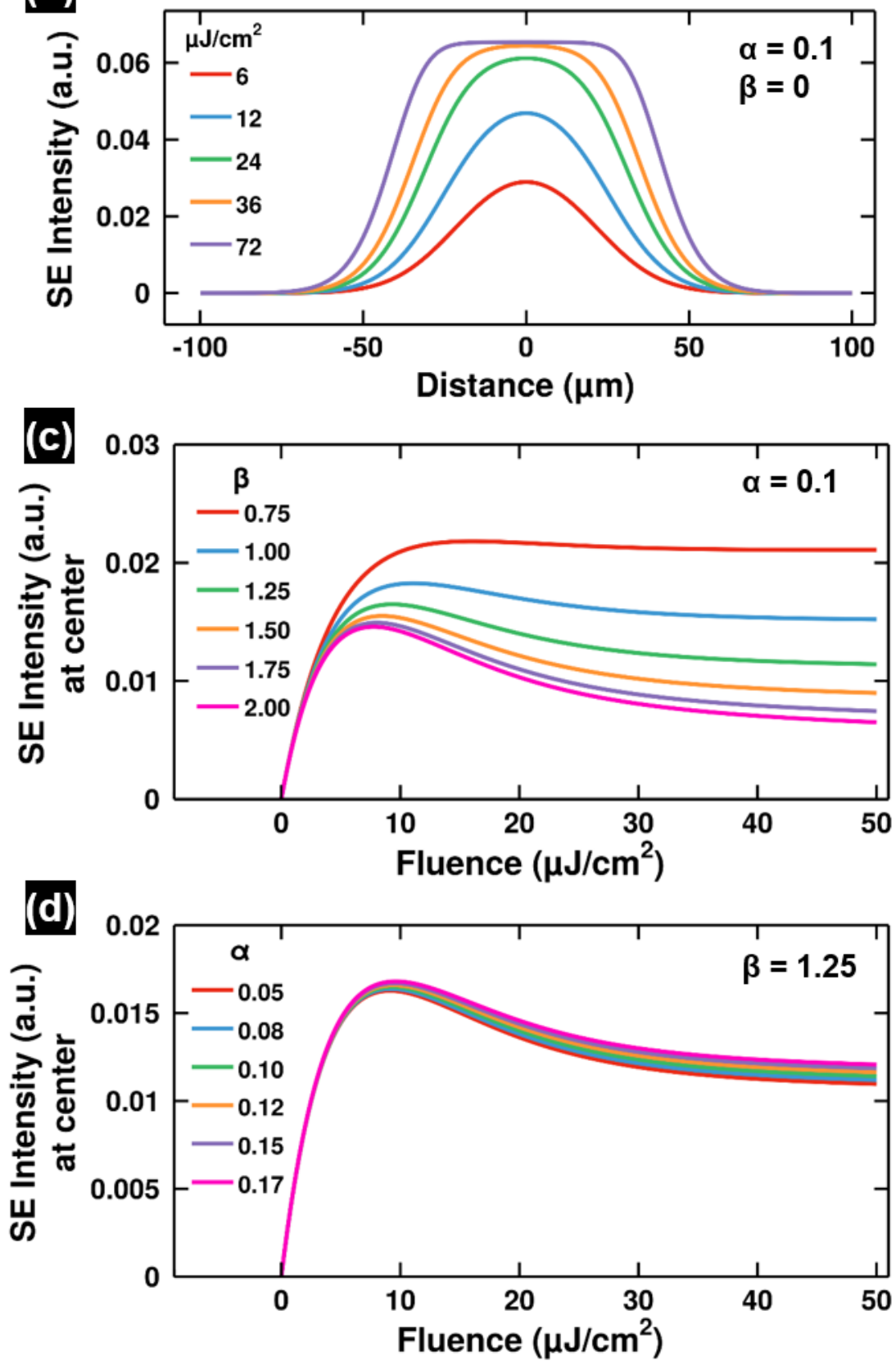
Fig. S6 Theoretical model results of fluence-dependent spatial intensity profiles and their behavior with two varying rates, $\alpha$ and $\beta$. (a,b) Line profiles at different excitation fluences with (a) $\alpha=0.1 \mathrm{ps}^{-1}$ and $\beta$ $=1.25 \mathrm{ps}^{-1}$, (b) $\alpha=0.1 \mathrm{ps}^{-1}$ and $\beta=0 \mathrm{ps}^{-1}$, respectively. Figure S6a shows the spatial distribution changes from a single Gaussian to a crater-shaped profile at sufficiently high excitation fluences, well capturing our experimental observation. As shown in Fig. S6(b), in the case of $\beta=0$, i.e., in the absence of a direct recombination channel from two e-h pair state to ground state, the spatial distribution at high fluences displays only a flat-top profile without an intensity dip at the center, indicating $\beta$ is crucial to give rise to crater-shaped features. (c,d) U-SEM image intensity at center spots as a function of excitation fluence: (c) at different $\beta$ values with $\alpha=0.1 \mathrm{ps}^{-1}$ and (d) at different $\alpha$ values with $\beta=1.25$ $\mathrm{ps}^{-1}$, respectively. The results indicate that the rate constant $\beta$ plays a key role in controlling cratershaped features [Fig. S6(c)], whereas $\alpha$ plays a minor role [Fig. S6(d)]. 

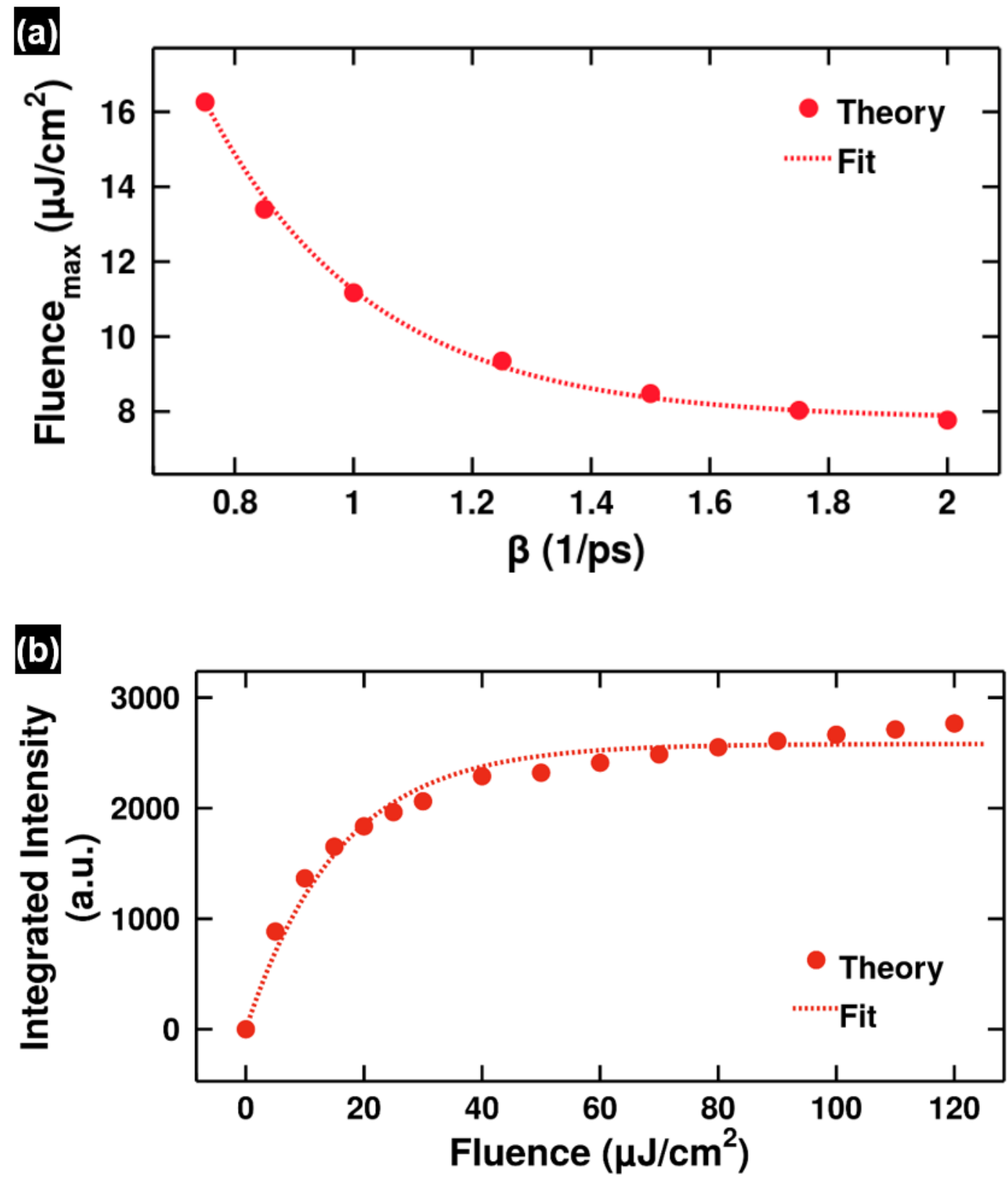

Fig. S7 Simulation results exhibiting a trend associated with the formation of crater-shaped features and the spatially integrated intensity which can be fitted approximately by a simple exponential function although the actual theoretical modeling involves complicated and lengthy mathematical expressions. (a) Maximum fluence (red filled circles), defined as the laser excitation fluence which starts to yield an intensity dip at the center spot (obtained from Fig. S6(b)), as a function of $\beta$. The red dotted line is a 
phenomenological fit to the simulation result, using $A \exp (-B \beta)+C$, where $A=126.0 \pm 21.6, B=3.6 \pm$ $0.2, C=7.8 \pm 0.1 .\left(\alpha=0.1 \mathrm{ps}^{-1}\right)$. (b) Integrated intensities as a function of laser excitation fluence (F). The red dotted line is a phenomenological fit to the simulation result, using $A[1-\exp (-F / B)]$, where $A$ $=2581.7 \pm 45.2, B=15.8 \pm 1.1\left(\alpha=0.1 \mathrm{ps}^{-1}, \beta=1.25 \mathrm{ps}^{-1}\right)$.

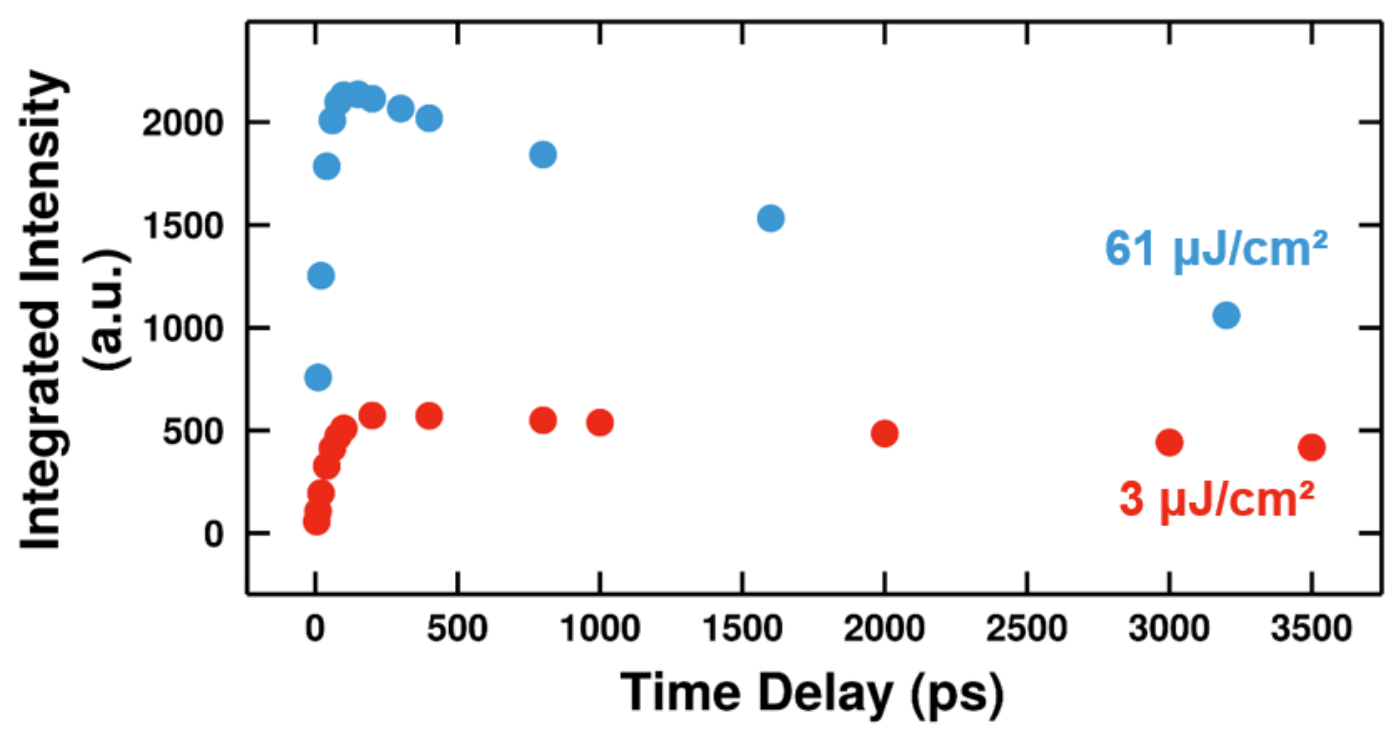

Fig. S8 Simulation result of intensities integrated within the field of view of the data shown in Fig. 2(b)-(c) at the pump excitation of 3 (red circles) and $60 \mu \mathrm{J} / \mathrm{cm}^{2}$ (blue circles) as a function of time delay. The simulated intensities capture the key temporal behavior seen in the experimental data [see Fig. 3(d)]. Note that for the simplified model presented here the zero time delay is defined as the moment when the pump laser excitation impinges onto a surface. 


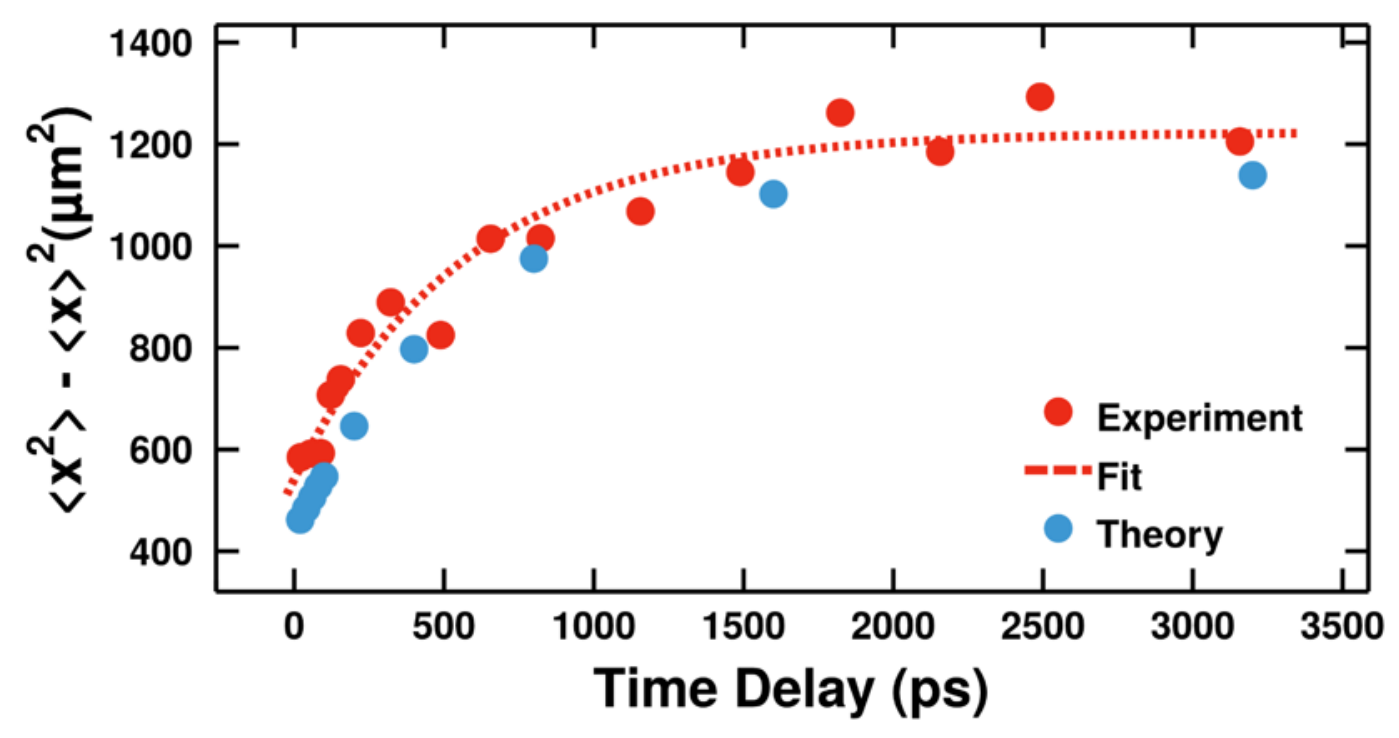

Fig. S9 Theoretical model results of mean square displacement (second moment) as a function of time delay at the laser excitation fluence of $3 \mu \mathrm{J} / \mathrm{cm}^{2}$. The red and blue circles indicate the experimental data and simulated result, respectively. The red dotted line is a fit to the experimental data, using $2 D_{0} \tau_{D}\left(1-\exp \left(-t / \tau_{D}\right)\right)$ 


\begin{tabular}{|c|l|c|}
\hline Parameter & \multicolumn{1}{|c|}{ Description } & Value \\
\hline$\alpha$ & $\begin{array}{l}\text { Decay rate of population from two e-h pair } \\
\text { state }\left(f_{2}\right) \text { to one e-h pair state }\left(f_{1}\right)\end{array}$ & $0.1 \mathrm{ps}^{-1}$ \\
\hline$\beta$ & $\begin{array}{l}\text { Decay rate of population from two e-h pair } \\
\text { state }\left(f_{2}\right) \text { to ground state }\left(f_{0}\right)\end{array}$ & $1.25 \mathrm{ps}^{-1}$ \\
\hline$D_{0}$ & Diffusion constant in the short-time limit & $0.65 \mu \mathrm{m}^{2} / \mathrm{ps}$ \\
\hline$\tau$ & Laser pulse width & $0.4 \mathrm{ps}$ \\
\hline
\end{tabular}

Table S1 The critical parameters used in our model to simulate the experimental results. Among these parameters, $\beta$ is the key one controlling the crater-shaped feature formation. $D_{0}$ is within the range of the experimentally extracted value [see text along with Fig. 3(c)]. The other, less important parameters, are $\gamma=0.00009 \mathrm{ps}^{-1}$ for electron-hole recombination rate to ground state; $1 / \tau_{e x}=0.018 \mathrm{ps}^{-1}$ for charge separation rate for U-SEM signal detection; $\tau_{D}=568 \mathrm{ps}$ for the time constant of time-dependent diffusion constant. 


\section{References}

1 P. N. Romanets, F. T. Vasko, Phys. Rev. B 2010, 81, 241411(R).

2 T. Ando, J. Phys. Soc. Jpn. 2006, 75, 074716.

3 A. C. Ferrari, J. C. Meyer, V. Scardaci, C. Casiraghi, M. Lazzeri, F. Mauri, S. Piscanec, D. Jiang, K. S. Novoselov, S. Roth, and A. K. Geim, Phys. Rev. Lett. 2006, 97, 187401.

4 M. S. Dresselhaus, A. Jorio, M. Hofmann, G. Dresselhaus, and R. Saito, Nano Lett. 2010, 10, 751.

5 A. Das, S. Pisana, B. Chakraborty, S. Piscanec, S. K. Saha, U. V. Waghmare, K. S. Novoselov, H. R. Krishnamurthy, A. K. Geim, A. C. Ferrari, and A. K. Sood, Nature Mater. 2008, 3, 210. 